Discrete Comput Geom 28:495-510 (2002)

DOI: $10.1007 / \mathrm{s} 00454-002-2884-3$

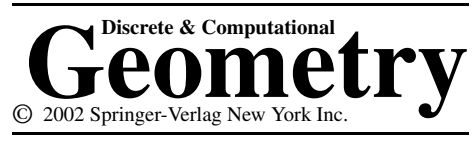

\title{
Combinatorial Geometry Problems in Pattern Recognition*
}

Peter Braß

Institute for Computer Science, Free University Berlin,

Takustraße 9, D-14195 Berlin, Germany

brass@inf.fu-berlin.de

\begin{abstract}
Combinatorial geometry problems motivated by point pattern matching algorithms are considered, and the classical exact matching situation and several variants are discussed.
\end{abstract}

\section{Introduction}

A connection of pattern recognition problems in the exact point pattern matching model to classical problems of combinatorial geometry was observed and used in a number of papers, e.g., [Bo], [AkTT], [Br], [BrK], [AgS] and [Br2]. Given a geometric pattern, and a background set in which to look for that pattern, an algorithm typically chooses some simple fragment of that pattern (just big enough to determine uniquely its extension to the full pattern) and first determines all occurrences of that fragment in the background. Then each of these possible occurrences is checked for the full pattern. So the maximum number of occurrences of that pattern fragment becomes an essential part of the time bound for any such algorithm.

A prototype for this situation is the congruent subset detection problem: given two sets $A, B$ of $m$ and $n$ points in the plane, find all subsets of $B$ that are congruent to $A$. Here the best known algorithm uses just two points as a fragment to identify the pattern. It chooses $a_{1}, a_{2} \in A$ arbitrarily, determines their distance and checks all those pairs $b_{1}, b_{2} \in B$ which have the same distance $d\left(b_{1}, b_{2}\right)=d\left(a_{1}, a_{2}\right)$ as possible image pairs. Each image pair $b_{1}, b_{2}$ determines a unique orientation-preserving congruence $\varphi$ mapping $a_{i} \mapsto b_{i}$, for which it remains to check on the full pattern set whether $\varphi(A) \subseteq B$. The unique orientation-reversing congruence determined by $b_{1}, b_{2}$ is handled similarly.

The complexity of this algorithm consists of two parts: the time needed to find all occurrences of that distance in $B$, and the number of occurrences found times $O(m \log n)$

* This research was supported by DFG Grant BR 1465/5-1. 
(the time needed to check whether $\varphi(A)$ is a subset of $B$ ). It is a famous open problem of Erdôs [Er] to bound the maximum number of occurrences of a (unit-)distance among $n$ points in the plane. The best known upper bound on the number of unit distance pairs is $O\left(n^{4 / 3}\right)$ [SpST], [ClEG ${ }^{+}$], [Sz], and they can be computed in $O\left(n^{4 / 3} \log n\right)$ time (similar to point-line incidences [Ma]). Thus the current bound for the complexity of this algorithm is $O\left(m n^{4 / 3} \log n\right)$, and any improvement in the combinatorial geometric bound for the number of unit distances problem will also improve the complexity bound of this pattern matching algorithm.

On the other hand, the combinatorial geometry lower bound gives, by its output complexity, a lower bound on the complexity of any algorithm solving the problem of listing all occurrences of the pattern. Thus the lattice section construction for sets with many unit distances also gives sets which contain $\Omega\left(n e^{c(\log n / \log \log n)}\right)$ congruent copies [Er] of some $m$-point pattern, at least for $m \ll n$. This illustrates the strong connection of the algorithmic problem and the combinatorial geometry problem.

The congruent subset detection problem described above is a special case of the following general situation: Given two sets $A$ (the pattern) and $B$ (the background) in $d$-dimensional space, and a group $\mathcal{G}_{d}$ (like translations, congruences, homotheties, similarities, affinities) that defines an equivalence relation on subsets of $d$-dimensional space, find all subsets of $B$ that are $\mathcal{G}_{d}$-equivalent to $A$.

In the following we discuss this problem, together with some variations. The group $\mathcal{G}_{d}$ will always be one of

- translations, $\operatorname{Transl}\left(E^{d}\right)$,

- homotheties, $\operatorname{Homoth}\left(E^{d}\right)$,

- orientation-preserving congruences, $\operatorname{Congr}^{+}\left(E^{d}\right)$,

- orientation-preserving similarities, $\operatorname{Simil}^{+}\left(E^{d}\right)$,

- affine maps, $\operatorname{Aff}\left(E^{d}\right)$.

The orientation-reversing part of each group can be added by running the algorithms a second time for the mirror-image of the pattern.

\section{Exact Matching: Basic Problems and the Number of Matches}

In the following $A$ will always denote the pattern set of $m$ points, and $B$ will always denote the background set of $n$ points $(m \leq n)$.

For each of the groups $\mathcal{G}_{d}$ above there is a number $c=c\left(\mathcal{G}_{d}\right)$ such that a $c$ tuple of image points determines a unique mapping from $\mathcal{G}_{d}\left(c\left(\operatorname{Transl}\left(E^{d}\right)\right)=1\right.$, $\left.c\left(\operatorname{Homoth}\left(E^{d}\right)\right)=2, c\left(\operatorname{Congr}^{+}\left(E^{d}\right)\right)=c\left(\operatorname{Simil}^{+}\left(E^{d}\right)\right)=d, c\left(\operatorname{Aff}\left(E^{d}\right)\right)=d+1\right)$. Thus there is always the algorithm that chooses arbitrarily $c$ points of $A$ and tests all $c$-tuples of points from $B$ as potential images; this needs $O\left(m n^{c} \log n\right)$ time. In the following we call this the trivial algorithm, and we compare all other algorithms against this.

The simplest case is that of translations in $d$-dimensional space $\mathcal{G}_{d}=\operatorname{Transl}\left(E^{d}\right)$. A translation is specified by the image of a single point, so the trivial algorithm needs $O(m n \log n)$ time. 
Proposition. It is possible to find all translates of an m-point set $A$ in an n-point set $B$ in d-dimensional space in $O(m n \log n)$ time.

There are many sets $B$ that have $\Omega(n)$ subsets that are translates of each other, e.g., $\{1, \ldots, n\}$ contains $n-m+1$ translates of $\{1, \ldots, m\}$. So if we want to list all subsets that are translates of the given pattern, this gives an output complexity of $m(n-m+1)$ (see also $[\mathrm{ReL}]$ ), and we really may need $\Omega(m n)$ time. However, these occurrences are completely specified if we just list the corresponding translation vectors. If we accept this as output, the output complexity is at most $n$, so there is hope for a faster algorithm.

If $T$ is the set of all translation vectors mapping $A$ into $B(t+A \subset B$ for each $t \in T)$, then $T+A \subset B$; so if there are sufficiently many matches $(|T| \gg n / m)$, then $T$ and $A$ are a set pair with a small Minkowski sum. This looks similar to Freiman's theorem (see p. $231 \mathrm{ff}$ of [Na]), so this should be possible only if $T, A$ and $B$ all have a "lattice-like" structure. Then, as in the case of the classical string-matching problem, a big number of matches is only possible if the sets have a very special structure, which could be used in an algorithm. This motivates

Conjecture 1. It is possible to find all $t$ with $t+A \subset B$ for sets $A, B$ of $m$ and $n$ real numbers in $O((m+n) \log n)$ time.

It should be mentioned that this problem has been discussed by a number of people, some of which believe the opposite, a lower bound near $\Omega(m n)$.

We can bound the maximum number of translates of a given pattern with much higher precision than is needed for the algorithmic application:

Theorem 1. The maximum number of translates of a fixed set $A \subset E^{d}$ among $n$ points in $E^{d}$ is $n-\Theta\left(n^{1-1 / k}\right)$, where $k$ is the dimension of the rational affine subspace spanned by $A$. The constant in the $\Theta$ depends on A.

The rational affine subspace spanned by $A$ is a translate of the rational linear subspace spanned by the difference set $A-A=\left\{a_{1}-a_{2} \mid a_{1}, a_{2} \in A\right\}$; the dimension of this space we call the rational dimension of the pattern. The rational dimension of a set is at least as large as the normal (real) dimension, but it can be larger. Figure 1 shows three examples of five-point patterns in the plane, of rational dimensions 2, 3, 4, and sets of $n$

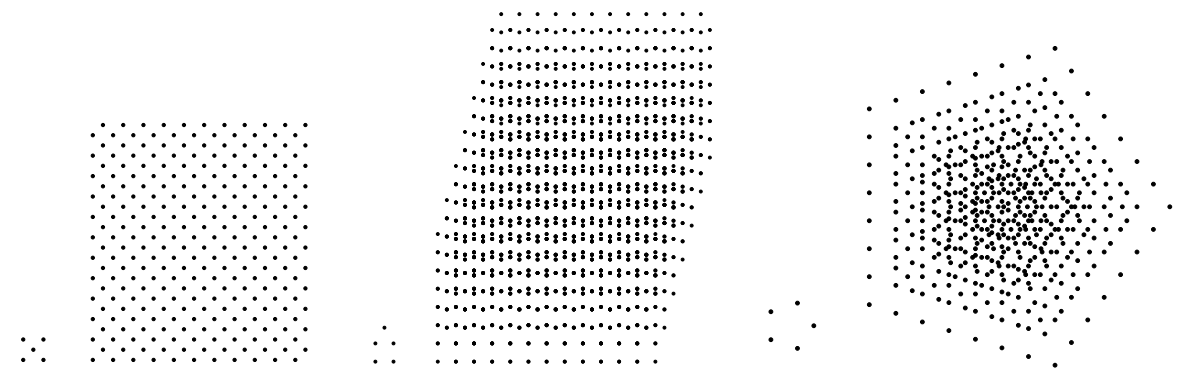

Fig. 1. Five-point patterns and sets with many translates. 
points containing $n-\Theta\left(n^{1 / 2}\right), n-\Theta\left(n^{2 / 3}\right)$ and $n-\Theta\left(n^{3 / 4}\right)$ translates of these patterns, respectively.

These sets are projections of lattice sections in a space whose dimension is that rational dimension of the pattern; so the second example is a projection of a three-dimensional lattice section. It also demonstrates an interesting phenomenon which happens for projections of $(d+1)$-dimensional lattice sections in $d$-dimensional space: there are only $O(1)$ different "types of neighbourhood" among the points. Depending on the interpretation of "types of neighbourhood", this leads to interesting higher-dimensional versions of the one-dimensional "three distances theorem" [Li], [Ch3] (for any $\alpha$ and $k$ there occur at most three distinct distances between successive elements of $\bigcup_{\kappa=0}^{k}(\mathbb{Z}+\kappa \alpha)$ ), as in [Ch1], [Ch2], where it is proved that for some affine image of the set there are only $O(1)$ incongruent Voronoi cells.

The next simplest case is that of homotheties $\mathcal{G}_{d}=\operatorname{Homoth}\left(E^{d}\right)$. A homothety is specified by a translation and a scale factor, so it is certainly determined by the images of two points. Thus there are less than $n^{2}$ matches, and the trivial algorithm needs $O\left(m n^{2} \log n\right)$ time. This is not optimal. The maximum number of homothetic copies of a pattern was determined by Elekes and Erdôs [ElE], the special case of axis-aligned hypercubes occurs already in $[\mathrm{KrB}]$.

Theorem [ElE]. The maximum number of homothetical copies of a pattern set $A \subset E^{d}$ among $n$ points in $E^{d}$ is $O\left(n^{1+1 / d^{\prime}}\right)$, where $d^{\prime}$ is the dimension of the affine hull of $A$ $\left(d^{\prime} \leq d\right)$. If $A$ can be written as set with algebraic coordinates with respect to some basis, then there is a matching lower bound, a construction with $\Omega\left(n^{1+1 / d^{\prime}}\right)$ homothetic copies among $n$ points, otherwise there is a lower bound of $\Omega\left(n^{1+1 / d^{\prime}-b(\log n)^{-a}}\right)$ for some $a, b>0$ depending on $A$.

Thus for full-dimensional pattern sets $A\left(d=d^{\prime}\right)$ the maximum number of homothetic copies among $n$ points is either $\Theta\left(n^{1+1 / d}\right)$ or slightly less (but still more than $c n^{1+1 / d-\varepsilon}$ for each $\varepsilon>0$ and sufficiently large $n$ ). It is also possible to find the points in that time:

Theorem 2. All homothetical copies of a fixed full-dimensional set $A \subset E^{d}$ of m points among an n-point set $B$ in $E^{d}$ can be found in $O\left(m n^{1+1 / d} \log n\right)$ time.

Again the special case of finding axis-aligned hypercubes was already solved in [KrB]. The complexity of finding homothetic copies becomes smaller with increasing dimension, a rare occurrence.

The geometrically most interesting case is that of congruences $\mathcal{G}_{d}=\operatorname{Congr}^{+}\left(E^{d}\right)$. Congruences in $d$-dimensional space are (up to orientation) determined by the image of one $(d-1)$-dimensional simplex. So the trivial algorithm takes $O\left(m n^{d} \log n\right)$, which can be improved on with better bounds on the number of congruent $(d-1)$-dimensional simplices among $n$ points in $E^{d}$ (an old question of Erdôs and Purdy [ErP]), and the time required to list these congruent simplices.

The two-dimensional case was treated in the Introduction:

Theorem. The maximum number of congruent copies of a set $A \subset E^{2}$ among $n$ points in $E^{2}$ is $O\left(n^{4 / 3}\right)$. All congruent copies of $A$ in $B$ can be found in $O\left(m n^{4 / 3} \log (n)\right)$ time. 
No progress seems possible here unless the upper bound for the maximum number of unit distances problem is improved.

For the three-dimensional problem there were several algorithms proposed [Bo], $[\mathrm{BoH}],[\mathrm{Br} 1]$, the currently best is implicit in $[\mathrm{AgS}]$.

Theorem [AgS]. It is possible to find all subsets of a set $B$ of $n$ points in threedimensional space that are congruent to a given set A of $m$ points in $O\left(m n^{5 / 3} \log (n)\right.$ $\left.2^{O\left((\alpha(n))^{2}\right)}\right)$ time, where $\alpha(n)$ is the inverse Ackermann function.

This three-dimensional result is complemented by an $\Omega\left(n^{4 / 3}\right)$ lower bound for the number of possible matches, a set of $n$ points in $E^{3}$ containing $c n^{4 / 3}$ triangles congruent to a given triangle ([AbF2], generalizing [ErHP]).

For higher dimensions there is only the $O\left(m n^{d}\right)$ algorithm of [ReL], a $(\log n)$-improvement of the trivial algorithm, and the bounds in $[\mathrm{AgS}]$ on the number of possible matches. The probably correct value for the number of matches was already conjectured by Erdős and Purdy [ErP]; it is achieved by a Lenz-type construction.

Conjecture 2. The maximum number of congruent $(d-1)$-dimensional simplices among $n$ points in $d$-dimensional space is $\Theta\left(n^{d / 2}\right)$ for $d \geq 4$ even, $\Theta\left(n^{(d-1) / 2+1 / 3}\right)$ for $d \geq 3$ odd. The extremal sets can be covered by $d / 2$ pairwise orthogonal planes $(d \geq 4$ even or $(d-3) / 2$ pairwise orthogonal planes and one three-dimensional subspace $(d \geq 3$ odd $)$. The simplices congruent to a given one among $n$ points in $d$-dimensional space can be listed in $O\left(n^{d / 2} \log n\right)$ time for $d \geq 4$ even, $O\left(n^{(d-1) / 2+1 / 3} \log n\right)$ time for $d \geq 3$ odd.

Also quite interesting is the case of similarities $\mathcal{G}_{d}=\operatorname{Simil}^{+}\left(E^{d}\right)$. A similarity in $d$-dimensional space is again determined (up to orientation) by the image of a $(d-1)$ simplex.

Proposition. The maximum number of similar copies of a set $A \subset E^{d}$ among $n$ points in $E^{d}$ is $O\left(n^{d}\right)$. All similar copies of $A$ in $B$ can be found in $O\left(m n^{d} \log n\right)$ time.

Unlike the case of congruent copies, the trivial algorithm is good at least in the planar case, for a set of $n$ points in the plane can contain $\Theta\left(n^{2}\right)$ similar triangles (e.g., a section of the triangular lattice contains by $(\pi / 3)$-rotation symmetry that many equilateral triangles). In fact the number of similar subsets of an $n$-point set in the plane was investigated in some detail [ElE], [LaR], [AbF]:

Theorem [ElE], [LaR]. The maximum number of similar copies of a set $X \subset E^{2}$, $|X| \geq 4$, among $n$ points in the plane is $\Theta\left(n^{2}\right)$ iff for every four points $x_{1}, x_{2}, x_{3}, x_{4} \in$ $X$ (the points interpreted as complex numbers) the cross-ratio $\left(\left(x_{3}-x_{1}\right)\left(x_{4}-x_{2}\right)\right) /$ $\left(\left(x_{4}-x_{1}\right)\left(x_{3}-x_{2}\right)\right)$ is algebraic; else it is o( $\left.n^{2}\right)$, but $\Omega\left(n^{\left.2-b(\log n)^{-a}\right)}\right)$, with $a, b>0$ depending on $X$. The maximum number of similar copies of a triangle $(|X|=3)$ is always $\Theta\left(n^{2}\right)$.

Unfortunately this result is very much dependent on the interpretation of the plane as complex numbers, and does not generalize to higher dimensions. For the three-dimensional 
situation Akutsu et al. [AkTT] give an $O\left(n^{2+1 / 5}\right)$ upper bound for the number of similar subsets, which considerably improves the $o\left(n^{3}\right)$ bound observed in [ElE]; but the lower bound is only the $\Omega\left(n^{2}\right)$ planar construction, and we do not have any full-dimensional construction giving a quadratic number of similar subsets. The best lower bound for similar copies of a full-dimensional pattern (not restricted to a plane) in three-dimensional space is $\Omega\left(n^{4 / 3}\right)$, which can be reached in two ways: by congruent copies alone [AbF], and by homothetic copies alone. No construction is known that takes advantage of the full group of similarities.

Conjecture 3. The maximum number of similar copies of a full-dimensional set $A \subset$ $E^{3}$ among $n$ points in $E^{3}$ is $o\left(n^{2}\right)$.

For higher dimensions, there is only the trivial $O\left(n^{d}\right)$ upper bound, and the $\Omega\left(n^{d / 2}\right)$ lower bound from the number of congruent subsets.

The case of affinities $\mathcal{G}_{d}=\operatorname{Aff}\left(E^{d}\right)$, finally, is simple: the output complexity almost reaches the bound of the trivial algorithm. Any affinity is determined by the image of a $d$-dimensional simplex, so the trivial algorithm needs $O\left(m n^{d+1} \log n\right)$; and there are pattern sets for which there are $\Theta\left(n^{d+1}\right)$ affine copies among $n$ points possible. For example, the $d$-dimensional lattice cube $\left\{1, \ldots, n^{1 / d}\right\}^{d}$ contains $\Theta\left(n^{d+1}\right)$ affine images of a small lattice cube (side length $O(1)$ ). Thus:

Proposition. The maximum number of affine copies of a set $A \subset E^{d}$ among $n$ points in $E^{d}$ is $O\left(n^{d+1}\right)$. This upper bound is reached for some sets $A$. All affine copies of $A$ in $B$ can be found in $O\left(m n^{d+1} \log n\right)$ time.

It would be interesting whether this order of $\Theta\left(n^{d+1}\right)$ affine images among $n$ points can be reached for each set $A$, or on what properties of $A$ this depends.

\section{Exact Point Pattern Matching with Preprocessing}

In all the algorithms above, the situation is that we are simultaneously given the pattern and the background, and have to identify the matches. There are two reasonable variants of this that include preprocessing:

- Given a fixed background, preprocess it some way such that one can decide quickly for each given pattern if and where it occurs.

- Given a fixed pattern, preprocess it some way such that one can decide quickly for each given background if and where it contains that pattern.

An example for the first setting would be the well-known "star matching" application: given a fixed star map, preprocess it some way so that we can identify the direction we are facing by looking at a picture of a part of the sky (pattern) (e.g., [WeKA]). An example for the second situation would be that we know how some traffic sign looks, and have to check a sequence of images taken from a car for occurrences of that sign.

In both situations, after preprocessing, a useful algorithm should be about linear in the size of the point set that forms each query, and only very small (logarithmic) in the 


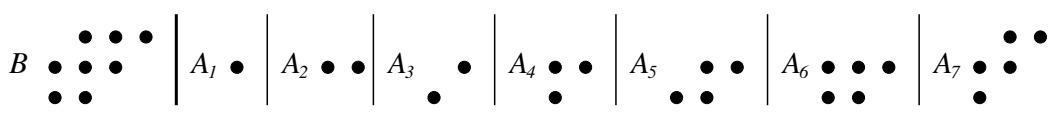

Fig. 2. A pattern $B$ and its maximally repeated subpatterns.

size of the point set that is preprocessed. In the second situation this is impossible: in the case of two-dimensional pattern matching under congruence, if we could solve that problem, we could specialize the pattern to two points and get an algorithm that finds unit distances in linear time. However, we have a superlinear lower bound for the problem of listing all unit distances by its output complexity. In the first situation, however, there is a data structure which solves this problem:

Theorem 3. For each choice of $d$ and $\mathcal{G}_{d}$ it is possible to preprocess a set $B \subset \mathbb{R}^{d}$ of $n$ points in such a way that for each query set $A$ of $m$ points it can be decided in $O(m \log n)$ time whether there is a subset of $B$ that is $\mathcal{G}_{d}$-equivalent to $A$, and all $\mathcal{G}_{d}$-equivalent subsets can be found in $O(m \log n+k)$ time if there are $k$ such subsets.

The preprocessing time of this algorithm depends on the number $r$ of maximally repeated patterns in $B$ defined below; for translations it is $O\left(r n^{2} \log n\right)$, and we conjecture $r=O(n)$.

Let $A_{\mathcal{G}_{d}}$ denote the class of all sets that are images of $A$ under $\mathcal{G}_{d}\left(\mathcal{G}_{d}\right.$-equivalent to $\left.A\right)$; a representative of the pattern is one element of that class. The pattern $A_{\mathcal{G}_{d}}$ is repeated in $B$ if there are several subsets of $B$ that are representatives of $A_{\mathcal{G}_{d}}$. For a repeated pattern there are $g_{1}, \ldots, g_{k} \in \mathcal{G}_{d}, k \geq 2$, such that $g_{i}(A) \subset B$. It is maximally repeated if $A=\bigcap_{i=1}^{k} g_{i}^{-1}(B)$, i.e., $A$ is the largest pattern occurring at these places. Figure 2 shows a set $B$ of eight points, and representatives of all maximally repeated patterns $A_{1}, \ldots, A_{7}$ occurring under translation in $B$.

The number of repeated patterns in an $n$-point set can of course be exponential, but we believe the number of maximally repeated patterns to be quite small. For example, in $\{1, \ldots, n\}$ under translations, any subset not containing both endpoints belongs to a repeated pattern, but the only maximally repeated patterns are the intervals $\{i, i+$ $1, \ldots, j\}$, because for any $t$ with $t+\{i, j\} \subset\{1, \ldots, n\}$ we already have $t+\{i, \ldots, j\} \subset$ $\{1, \ldots, n\}$. So there are only $n-1$ maximally repeated patterns.

Conjecture 4. The total number of maximally repeated patterns in an n-point set under translations is $O(n)$. The total number of maximally repeated patterns in an n-point set under congruences is $O\left(n^{d}\right)$.

\section{Window Matching and Approximate Matching}

All algorithms mentioned above were for the model of exact pattern matching, which is of course of purely theoretical interest: real-world data and real-world computation always involve errors. So it would be reasonable to allow some error for the matches, looking for subsets $B^{\prime} \subseteq B$ that are perhaps not equal to $g(A)$ for some $g \in \mathcal{G}_{d}$, but 


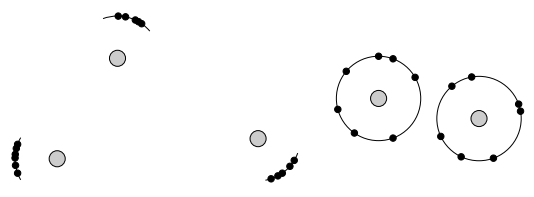

Fig. 3. A pattern and a set with many optimal matches.

have at least a small distance: $d\left(B^{\prime}, g(A)\right) \leq \varepsilon$. The most natural distance measure is the Hausdorff-distance

$$
d_{\text {Hausdoff }}(X, Y)=\max \left(\max _{x \in X} \min _{y \in Y} d(x, y), \max _{y \in Y} \min _{x \in X} d(x, y)\right) .
$$

So one should look for subsets $B^{\prime} \subseteq B$ and transformations $g \in \mathcal{G}_{d}$ that minimize $d_{\text {Hausdorff }}\left(B^{\prime}, g(A)\right)$. This Hausdorff-approximate pattern matching has also been much studied [AlG], but is, for practical applications, similarly useless, since the algorithms there involve such high complexities as to make them quite impracticable. Thus the best known algorithm for finding the optimum Hausdorff-approximately congruent copy of an $m$-point set in an $n$-point set in the plane has a complexity of $O\left((n+m)^{6} \log (n m)\right)$ $[\mathrm{HuKK}],[\mathrm{AlG}]$. Also there is a big difference with the exact matching setting in that we cannot expect to get all matches, not even all optimal matches, since there can be exponentially many of them. To see this, we choose the pattern set $A$ fixed, and around each point of $A$ a circle of radius $\varepsilon$, which must be smaller than one-third of the minimum distance between points of $A$. Then we construct $B$ by choosing the $n$ points in $m$ groups of $n / m$ on each of these circles, taking care that for three points $a_{1}, a_{2}, a_{3} \in A$ the points are concentrated on a short arc of the circle such that any movement of $A$ will move at least one of these points away from its group (Fig. 3). One possibility for such a choice is to take for $a_{1}$ the angle interval ]0, $\frac{1}{3} \pi$ [, for $a_{2}$ the angle interval ] $\frac{2}{3} \pi, \pi$ [ and for $a_{3}$ the angle interval ] $\frac{4}{3} \pi, \frac{5}{3} \pi$ [; then for each direction $w$ there is an $i$ with the angle between $w$ and any vector in the interval is larger than $\pi / 2$, so a translation in direction $w$ increases the distance to any point on the arc around $a_{i}$. Similar constructions work also for congruences. Thus each choice of one point from each group gives an error $\varepsilon$, and there is no subset with a smaller error.

So a direct translation of the questions of the exact matching model is useless, since it would force exponential output complexity. One version to avoid this is to work instead in the transformation space, and describe the set of all elements of $\mathcal{G}_{d}$ that maps $A$ within Hausdorff-distance $\varepsilon$ of a subset of $B$. This is a very complete description of all possible matches, but it still involves a very high (although still polynomial) complexity. Lower bounds for the case of translations were constructed in [Ru].

A different way to avoid this problem uses the fact that such constructions giving many optimal matches need many points in $B$ that can be interchangeably matched to the same points of $A$; so these points in the background set have to be very close together, with a distance smaller than the Hausdorff error bound $\varepsilon$. So one can avoid this problem by introducing a lower bound on the smallest distance in $B$, e.g., by considering only integer-coordinate patterns, or by introducing the smallest distance in $B$ as an additional 
parameter, or by discretizing the set to some suitably scaled lattice. In the last case one can obtain only approximate solutions, since one does not distinguish points with the same discretization, but one avoids the exponential number of matches, since in the discretized subset there are only a few correct matches possible. So in this setting the problems can indeed be solved quickly $[\mathrm{CaS}],[\mathrm{InV}]$, but they become incomparable with the original Hausdorff-approximate pattern matching, losing much of the geometrically interesting problems, and introducing many further parameters on which the complexity depends (the diameter of the sets, the Hausdorff error bound $\varepsilon$, the approximation error for the approximate solutions). In any such setting based on discretization, we cannot have the exact matching problem $\varepsilon=0$ as special case of the approximate matching; for the complexity bound becomes infinite for $\varepsilon \rightarrow 0$.

In this section a variant is proposed which avoids the difficulty of potentially exponential many matches while essentially retaining the Hausdorff-approximate matching model. This variant leads, even in the exact model, to interesting open problems.

Let again $A$ (pattern) and $B$ (background) be sets of $m$ and $n$ points in the plane. The idea of "window-matching" is not to look for arbitrary subsets of $B$ that are congruent to $A$, but to look for sections cut out of $B$ by a convex set $W$ (the window). A subset $B^{\prime} \subseteq B$ is a window-match of $A$ if

- $B^{\prime}$ is congruent (or approximately congruent) to $A$, and

- $B^{\prime}=B \cap W$.

This is a very reasonable assumption, e.g., in the "star-matching" problem [WeKA], and, depending on the nature of the window, it gives interesting restrictions for the number of matches. The smallest possible window is the convex hull of the points in the matching subset: we can ask for the number of subsets $B^{\prime} \subset B$ such that $B^{\prime}$ is congruent to $A$ and $\operatorname{conv}\left(B^{\prime}\right) \cap B=B^{\prime}$. This already leads in the smallest nontrivial case ( $A$ a triangle) to an open problem:

Conjecture 5. Any set of $n$ points in the plane contains only $O(n)$ empty congruent triangles.

A slightly weaker statement would be that for each triangle $\Delta$ there are at most $c(\Delta) n$ empty congruent copies of $\Delta$ among $n$ points in the plane. Both conditions are really necessary here: there are sets of $n$ points with $\left(\begin{array}{l}n \\ 3\end{array}\right)$ empty triangles, and sets with $\Omega\left(n e^{(c \log n) / \log \log n}\right)$ congruent triangles.

The same statement trivially holds for translations, since there are less than $n$ translates of any set $A$, even without the emptiness condition. It also holds for homothetic copies: in a set $B$ of $n$ points in the plane, there are less than $n$ or less than $2 n$ empty homothetic copies of a given triangle $x y z$, depending on whether we allow additional points on the boundary of the convex hull of the set while still accepting it as "empty". A homothetic copy is specified by the image points of $x y$, so if we partition $B$ by lines parallel to $x y$, then the homothetic copies are specified by intervals on such lines with endpoints in $B$. However, if two such intervals share a left endpoint, then the larger triangle has another point on its boundary; and if one interval is contained in the interior of another interval, then the larger triangle has an additional interior point. Thus $k$ points of $B$ on such a line specify at most $k-1$ or $2 k-3$ homothetic triangles without violating the weak or strong 
emptyness condition, respectively. The conjecture may even hold for similar copies, but it obviously fails for affine copies, since any two triangles are affine equivalent.

Another natural choice for the window is a circular disc; in that case the bound on the number of occurrences becomes simple:

Theorem 4. In a set $B$ of $n$ points in the plane there are $O(n)$ subsets $B^{\prime}$ such that $B^{\prime}$ is a translate, homothetic, congruent or similar copy of a given set A of $m$ points, $1 \leq m \leq n$, and $B^{\prime}=B \cap D$ for some circular disc $D$.

However, although a list of possible matches can be computed fast (the list of mappings in $O((m+n) \log n))$, it is, different from the subset-matching situation discussed in the beginning of this paper, not obvious how to verify that they are really window matches, cut out by a circular disc.

For the approximate matching situation, we have to make sure that the window is slightly larger than the convex hull of the matching subset, so that each pattern point is well inside the window; otherwise it is simple to construct sets with an exponential number of matches.

Conjecture 6. There is a $c$ such that, for each $\varepsilon \geq 0$ and all sets $A, B,|B|=n$, there are less than cn subsets $B^{\prime} \subset B$ with the properties

- $d_{\text {Hausdorff }}\left(B^{\prime}, A^{\prime}\right) \leq \varepsilon$ for some congruent copy $A^{\prime}$ of $A$, and

- $B^{\prime}=B \cap\left(\operatorname{conv}\left(B^{\prime}\right)+D_{\varepsilon}(0)\right)$.

Here $\left(\operatorname{conv}\left(B^{\prime}\right)+D_{\varepsilon}(0)\right)$ is the $\varepsilon$-neighbourhood of the convex hull of $B^{\prime}$ : the window.

\section{Proof of the Theorems}

Proof of Theorem 1. Let $A$ be the fixed pattern set, translated such that $0 \in A$. To prove the upper bound, we can restrict ourselves to a subset of $A^{\prime} \subseteq A$ consisting of rational independent vectors, since any occurrence of the full pattern set contains an occurrence of that subset. Let $A^{\prime}=\left\{0, a_{1}, \ldots, a_{k}\right\}$ where $a_{1}, \ldots, a_{k}$ are rationally independent. Let $B$ be a set of $n$ points that contains a maximum number of translates of $A^{\prime}$. Consider a graph on $B$, joining two vertices $b_{1}, b_{2} \in B$ by an edge marked $j$ iff $b_{2}-b_{1}=a_{j}$ for some $j$. This graph can be assumed to be connected; if $B$ consists of several connected components, we can translate them independently, without changing the number of translates of $A^{\prime}$ contained in that set, until the number of connected components decreases. So any two points $b_{1}, b_{2} \in B$ are joined by such a path; thus their difference $b_{2}-b_{1}$ is some integer combination of $a_{1}, \ldots, a_{k}$, and, by the assumption of rational independence, the coefficients of that combination do not depend on the path, but are determined by $b_{2}-b_{1}$. Keeping one point of $B$ fixed, we can thus assign $k$-dimensional integer coordinates to the points of $B$, where translates of $A^{\prime}=\left\{0, a_{1}, \ldots, a_{k}\right\}$ correspond to translates of the standard basis $\left\{0, e_{1}, \ldots, e_{k}\right\}$. Let $\hat{B}$ be this set of $n$ points in the $k$-dimensional integer lattice, and consider the cube packing $\hat{B}+\left[-\frac{1}{2}, \frac{1}{2}\right]^{d}$; this is a set of volume $n$ and has by the isoperimetric inequality [Ta] a surface of order $\Omega\left(n^{1-1 / k}\right)$. This surface consists of unit $(d-1)$-cubes with outer normals $\pm e_{i}$, which occur in equal number with both signs. 
Each face with outer normal $+e_{i}$ corresponds to a point $\hat{b}$ in $\hat{B}$, for which the translate $b+A^{\prime}$ is not contained in $B$ : for the point $b+a_{i}$ is missing from $B$, otherwise that face would be in the interior of the cube packing. By the same argument, each point $b \in B$ for which the translate $b+A^{\prime}$ is not a subset of $B$ contributes at least one and at most $k$ such faces (with outer normal $e_{i}$ if the point $b+a_{i}$ is missing from $B$ ) to the surface of the packing. Thus there are at least $\Omega\left(n^{1-1 / k}\right)$ points $b \in B$ for which $b+A^{\prime} \not \subset B$, so the number of occurrences of translates of $A$ in $B$ is at most $n-\Omega\left(n^{1-1 / k}\right)$. To prove the lower bound, we argue as follows: if $A=\left\{0, a_{1}, \ldots, a_{m-1}\right\}$ spans a rational subspace of dimension $k$, then there are $k$ vectors $v_{1}, \ldots, v_{k}$ such that each element of $A$ is an integer linear combination of these $v_{i}$. Let $c=c(A)$ be the maximum absolute value of a coefficient needed by these integer combinations; then the set

$$
\left\{\sum_{i=1}^{k} \alpha_{i} v_{i} \mid \alpha_{i} \in\left\{1, \ldots, n^{1 / k}\right\} \text { for } i \in\{1, \ldots, k\}\right\}
$$

is a set of $n$ points that contains at least $\left(n^{1 / k}-2 c\right)^{k}=n-O\left(n^{1-1 / k}\right)$ points $p$ with all $\alpha_{i} \in\left\{1+c(A), \ldots, n^{1 / k}-c(A)\right\}$, and for any such point $p$ the whole translate $p+A$ still belongs to that set of $n$ points. So there are $n-O\left(n^{1-1 / k}\right)$ translates of $A$ in that set.

The dependence of the multiplicative constant in the $O\left(n^{1-1 / k}\right)$ on $A$ is unavoidable: for the pattern $\{0,1, n\}$ any set of $n$ points will contain less than $\frac{1}{2} n$ translates of that pattern.

Proof of Theorem 2. The algorithm is very simple: Let $A$ be the given pattern and let $B$ be the set of points in which we are looking for homothetic copies of $A$.

0 . Renumber the elements of $A$ such that $a_{1}, a_{2}, \ldots, a_{d+1}$ are affinely independent.

1. For $i=2, \ldots, d+1$

1.1. Construct for each point $b_{j} \in B$ the line $\left\{b_{j}+t\left(a_{i}-a_{1}\right) \mid t \in R\right\}$ (in direction $a_{i}-a_{1}$ ), and collect for each constructed line all points of $B$ giving the same line. Label each constructed line by the number of points of $B$ it contains.

2. For each point $b \in B$

2.1. Find the line $\left\{b+t\left(a_{i}-a_{1}\right) \mid t \in R\right\}$ through $b$ in one of the directions $a_{i}-a_{1}$ which contains the fewest points of $B$.

2.1.1. For each point $b^{\prime} \in B, b^{\prime} \neq b$ on that line, construct the homothety $h$ which maps $a_{1}$ to $b$ and $a_{i}$ to $b^{\prime}$, and test whether $h(A) \subset B$.

The correctness of the algorithm above is obvious: if there is a homothetical copy of $A$ in $B$ with $b$ as an image of $a_{1}$, then for each $i$ the image of $a_{i}$ must be on the line $\left\{b+t\left(a_{i}-a_{1}\right) \mid t \in R\right\}$; and the image of two points completely specifies the homothety.

It remains to bound the complexity. For a fixed dimension, all steps but 2.1.1 can be executed in $O(n \log n)$ time, so we have only to bound the number of point pairs $b, b^{\prime}$, for which in step 2.1.1 the homothety $h$ is constructed and tested, which takes $O(m \log n)$ time per pair $b, b^{\prime}$. For this we have to show that for most points $b$ there is one direction in which the line through that point contains few other points $b^{\prime}$ of the set.

We can take the $d$ linear independent directions $a_{2}-a_{1}, \ldots, a_{d+1}-a_{1}$ as coordinate axes of our space; then point $b^{\prime}$ is in the direction of the $i$ th line of $b$ iff all coordinates of $b$ and $b^{\prime}$ (relative to that coordinate system) with the only exception of the $i$ th coordinate 
agree. So $B$ can be interpreted as a set of $d$-tuples of some cartesian product $B \subset X^{d}$ (where $X$ in this application is $\mathbb{R}$, but this is not needed), and the number of point pairs $b, b^{\prime}$ that are tried in step 2.1.1 of the algorithm is

$$
\sum_{b \in B} \min _{i=1, \ldots, d} \mid\left\{b^{\prime} \in B \mid b^{\prime} \text { differs from } b \text { only in the } i \text { th coordinate }\right\} \mid .
$$

That is, for each point $b=\left(x_{1}, \ldots, x_{d}\right)$ we count the number of $\left(x_{1}^{\prime}, \ldots, x_{d}^{\prime}\right) \in B$ with $x_{j}^{\prime}=x_{j}$ for all $j \neq i$, taking the minimum over all $i$. Let $f_{d}(n)$ be the maximum over all $n$-element sets of $d$-tuples of that sum:

$$
f_{d}(n)=\max _{\substack{B \subset X^{d} \\|B|=n}} \sum_{\left(x_{1}, \ldots, x_{d}\right) \in B} \min _{i=1, \ldots, d} \#\left\{\left(x_{1}^{\prime}, \ldots, x_{d}^{\prime}\right) \in B \text { with } x_{j}^{\prime}=x_{j} \text { for all } j \neq i\right\},
$$

then the claim of Theorem 2 follows from the bound

Lemma. $\quad f_{d}(n) \leq n^{1+1 / d}$ with equality if $n=m^{d}$ for $m \in \mathbb{N}$.

This lemma can also be read from the proof of the corresponding theorem in [ElE]; since it is a purely combinatorial statement of possibly independent interest, it is given here with a full proof.

Proof of the Lemma. Equality can be reached if $B$ is a full cartesian product, $B=Y^{d}$ for some set $Y \subset X,|Y|=m$, because, for each $\left(y_{1}, \ldots, y_{d}\right) \in Y^{d}$ and each $i$, the number of $\left(y_{1}^{\prime}, \ldots, y_{d}^{\prime}\right) \in Y^{d}$ with $y_{j}^{\prime}=y_{j}$ for all $j \neq i$ is $m$.

The proof of the upper bound is by induction on $d$, the claim being trivial for $d=1$. Let $d \geq 2$ and let $B$ be an extremal set with $n$ elements. Let $z_{1}, \ldots, z_{k}$ be the distinct elements of $X$ that occur as the last coordinate of some $b \in B$; then $B_{\kappa}=\left\{\left(x_{1}, \ldots, x_{d}\right) \in\right.$ $\left.B \mid x_{d}=z_{\kappa}\right\}$ is a partition of $B$, and

$$
f_{d}(n)=\sum_{\kappa=1}^{k} \sum_{\left(x_{1}, \ldots, x_{d}\right) \in B_{\kappa}} \min _{i=1, \ldots, d} \#\left\{\left(x_{1}^{\prime}, \ldots, x_{d}^{\prime}\right) \in B \text { with } x_{j}^{\prime}=x_{j} \text { for all } j \neq i\right\} .
$$

However, for the contribution of each class $B_{\kappa}$ there are two simple upper bounds:

- If we restrict the minimum over the coordinates to the first $d-1$ coordinates $(i \neq d)$, then for each $\left(x_{1}, \ldots, x_{d}\right) \in B_{\kappa}$ all $\left(x_{1}^{\prime}, \ldots, x_{d}^{\prime}\right) \in B$ with $x_{j}^{\prime}=x_{j}$ for all $j \neq i$ will have $x_{d}^{\prime}=x_{d}$, so belong to the same set $B_{\kappa}$. So

$$
\sum_{\left(x_{1}, \ldots, x_{d}\right) \in B_{\kappa}} \min _{i=1, \ldots, d} \#\left\{\left(x_{1}^{\prime}, \ldots, x_{d}^{\prime}\right) \in B \text { with } x_{j}^{\prime}=x_{j} \text { for all } j \neq i\right\} \leq f_{d-1}\left(\left|B_{\kappa}\right|\right) .
$$

- If we restrict the minimum over the coordinates only to the last coordinate $(i=d)$, then the sets $\left\{\left(x_{1}^{\prime}, \ldots, x_{d}^{\prime}\right) \in B\right.$ with $x_{j}^{\prime}=x_{j}$ for all $\left.j \neq i\right\}$ for each $\left(x_{1}, \ldots, x_{d}\right) \in$ $B_{\kappa}$ will be disjoint, so the sum of their cardinalities is at most $n$ :

$$
\sum_{\left(x_{1}, \ldots, x_{d}\right) \in B_{\kappa}} \min _{i=1, \ldots, d} \#\left\{\left(x_{1}^{\prime}, \ldots, x_{d}^{\prime}\right) \in B \text { with } x_{j}^{\prime}=x_{j} \text { for all } j \neq i\right\} \leq n .
$$


Thus

$$
\begin{aligned}
f_{d}(n) & \leq \sum_{\kappa=1}^{k} \min \left(n, f_{d-1}\left(\left|B_{\kappa}\right|\right)\right) \\
& \leq \max _{\substack{\beta_{1}, \ldots, \beta_{k} \geq 1 \\
\beta_{1}+\cdots+\beta_{k}=n}} \sum_{\kappa=1}^{k} \min \left(n, f_{d-1}\left(\beta_{\kappa}\right)\right) \leq \max _{\substack{\beta_{1}, \ldots, \beta_{k} \geq 1 \\
\beta_{1}+\cdots+\beta_{k}=n}} \sum_{\kappa=1}^{k} \min \left(n, \beta_{\kappa}^{d /(d-1)}\right) \\
& \leq \max _{\substack{1 \leq \beta_{1}, \ldots, \ldots \beta_{k} \leq n \\
\beta_{1}+\cdots+\beta_{k}=n}} \sum_{\kappa=1}^{k} \beta_{\kappa}^{d /(d-1)} \leq \frac{n}{n^{(d-1) / d}}\left(n^{(d-1) / d}\right)^{d /(d-1)}=n^{(d+1) / d} .
\end{aligned}
$$

This is the claimed upper bound on $f_{d}(n)$, which completes the proof of the lemma and Theorem 2.

Proof of Theorem 3. Let $B$ be the given set of $n$ points, and let $\mathcal{G}_{d}$ the group generating the equivalence relation. We wish to preprocess $B$ in such a way that we can answer queries "is $A$ equivalent under $\mathcal{G}_{d}$ to a subset of $B$ ?" quickly. We do this by listing important subpatterns of $B$ (the maximally repeated patterns in $B$ ), and for each such subpattern the possible extensions, with pointers to the larger subpatterns determined by it. Then the query is processed by identifying increasing parts of $A$ in these possible subpatterns, keeping track of the possible occurrences in $B$ and the $\mathcal{G}_{d}$-mappings involved. An occurrence of $A$ in $B$ is completely described by the transformation with $g(A) \subset B$. We first describe the procedure for the case of translations.

Let $A_{1}^{\max }, \ldots, A_{r}^{\max }$ be a list of representatives of each maximally repeated pattern in $B$. An important property is that each repeated pattern is a subpattern of a uniquely determined smallest maximally repeated pattern, and this can be determined easily: if a pattern $A^{*}$ occurs in $B$ at the places $g_{1}^{*}\left(A^{*}\right), \ldots, g_{J}^{*}\left(A^{*}\right)$, then $\bigcap_{j=1}^{J} g_{j}^{*-1}(B)$ is a representative of the smallest maximally repeated pattern containing $A^{*}$.

Let $g_{1, i}, \ldots, g_{a_{i}, i} \in \mathcal{G}_{d}$ be the transformations giving the occurrences of the maximally repeated subpattern $A_{i}^{\max }$ in $B$, i.e., $g_{j, i}\left(A_{i}^{\max }\right) \subset B$, and also $A_{i}^{\max }=\bigcap_{j=1}^{a_{i}} g_{j, i}^{-1}$ (B). Construct $Z_{i}=\bigcup_{j=1}^{a_{i}} g_{j, i}^{-1}(B)$, and label each point $z \in Z_{i}$ by the list of all $g_{j, i}$ with $z \in g_{j, i}^{-1}(B)$ as references to the occurrences of that point.

Now $Z_{i}$ is the set of all possible extensions of $A_{i}^{\text {max }}$ that can occur in a subpattern of $B$. We now attach several further labels to these points. For each point $z \in Z_{i}$ we have either

- $z$ occurs for only one $j$ in $g_{j, i}^{-1}(B)$. Then the occurrence of $A_{i}^{\max } \cup\{z\}$ is uniquely determined; set $z$.status as "unique".

- $z$ occurs for several $j$ in $g_{j, i}^{-1}(B)$. Then $A_{i}^{\max } \cup\{z\}$ is a repeated pattern, so it is part of a smallest maximally repeated pattern $A_{k}^{\max }$ for some $k\left(A_{k}^{\max }=\bigcap\left\{g_{j, i}^{-1}(B) \mid\right.\right.$ $\left.z \in g_{j, i}^{-1}(B)\right\}$, possibly $\left.A_{k}^{\max }=A_{i}^{\max }\right)$. Then there is a function $h \in \mathcal{G}_{d}$ with $h\left(A_{i}^{\max } \cup\{z\}\right) \subset A_{k}^{\max }$. Set $z$.status as "ambiguous", $z$.next $=k$ and $z$.map $=h$.

To answer a query now, given a set $A=A^{\text {query }}=\left\{a_{1}, \ldots, a_{m}\right\}$, we determine the smallest maximally repeated patterns $A_{i_{\mu}}^{\max }$ containing $A_{\mu}^{\text {partial query }}=\left\{a_{1}, \ldots, a_{\mu}\right\}$ for $\mu=1, \ldots, m$. A single point is certainly a repeated pattern in $B$, even a maximally 
repeated pattern, since $B$ is finite, so one of the $A_{i}^{\max }$, we can assume $A_{1}^{\max }$, consists of a single point. The query algorithm for translations is as follows:

- Let $\gamma_{1}$ denote the translation that maps $A_{1}^{\text {partial query }}$ to $A_{1}^{\max }$, and set $\mu=1$, and $i_{1}=1$.

- For $\mu<m$ do

- Compute $\gamma_{\mu}\left(a_{\mu+1}\right)$, and check whether it is an element of $Z_{i_{\mu}}$.

- If $\gamma_{\mu}\left(a_{\mu+1}\right)$ does not occur in $Z_{i_{\mu}}$, stop: the pattern $A^{\text {query }}$ does not occur in $B$.

- If $\gamma_{\mu}\left(a_{\mu+1}\right)$ occurs in $Z_{i_{\mu}}$, and is labelled there as "unique", set $\gamma_{\mu+1}=g_{j, i_{\mu}} \circ \gamma_{\mu}$, where $g_{j, i_{\mu}}$ is the unique reference to the occurrence of that point in $B$. Check whether $\gamma_{\mu+1}\left(A^{\text {query }}\right)$ is a subset of $B$, and give the answer of this as the result.

- If $\gamma_{\mu}\left(a_{\mu+1}\right)$ occurs in $Z_{i_{\mu}}$, and is labelled there as "ambiguous", set $i_{\mu+1}=$ $\gamma_{\mu}\left(a_{\mu+1}\right)$.next and $\gamma_{\mu+1}=\left(\gamma_{\mu}\left(a_{\mu+1}\right)\right.$.map $) \circ \gamma_{\mu}$.

- If $\mu=m$, output $g_{j, i_{m}} \circ \gamma_{\mu}$, for $j=1, \ldots, a_{i_{m}}$ as the list of all occurrences of $A^{\text {query }}$

in $B$. (We give the transformations as output to keep output complexity small.)

This query algorithm requires at most $m$ steps (one for each point of $A^{\text {query }}$ that is identified), and each step requires only some computations of mappings (in $\mathcal{G}_{d}$ ) and one point location operation, which can be done in $O(\log n)$, so the total query time is $O(m \log n)$ plus the time for the output of all matches, if we need them all.

The time needed to build this structure is essentially determined by the number $r$ of maximally repeated patterns times the maximum size of all of the $Z_{i}$ structures, which for translations is $O\left(n^{2}\right)$, so for translations this time is $O\left(r n^{2} \log n\right)$.

The one difference for other groups $\mathcal{G}_{d}$ is that we need to begin with patterns big enough that a mapping from $\mathcal{G}_{d}$ is determined by its values on that pattern. Thus a single point, as used as the starting configuration for the translations, is of course also a repeated pattern for congruences, but there are infinitely many congruences mapping that point on a point of $B$. To make the structure above work, we need instead to start with a sufficiently high-dimensional simplex in our query pattern that uniquely defines its extension by a $\mathcal{G}_{d}$-mapping ( $c(G)$ points, as discussed in Section 2, in the trivial algorithm). We have to bring each of these $O\left(n^{c\left(\mathcal{G}_{d}\right)}\right)$ simplices in a normalized position, and collect for each of them the occurrences of that simplex in $B$ represented by the group operations $g \in \mathcal{G}_{d}$ mapping that canonical-position simplex into $B$. For each of these starting simplices we now have to construct the possible extensions, the $Z_{i}$-structure. The size of each $Z_{i}$ structure is at most $n$ times the maximum number of occurrences of a pattern in $B$ (the function discussed in Section 2), and for each of those $n^{c\left(\mathcal{G}_{d}\right)}$ starting simplices there are at most $r$ such structures to be built, where $r$ is again the number of maximally repeated subsets. Using the trivial bound on the number of occurrences of a pattern, this gives a rough upper bound of $r n^{2 c\left(\mathcal{G}_{d}\right)+1}$ for the total size of the structure, which can be built in $r n^{2 c\left(\mathcal{G}_{d}\right)+1} \log n$ time.

For the query algorithm we start with an arbitrary $\left(c\left(\mathcal{G}_{d}\right)-1\right)$-dimensional simplex in $A^{\text {query }}$, bring it in some normalized position (in $O(1)$ time) so that it can be decided whether that simplex is among those occurring in $B$, and if it occurs several times, follow the algorithm above, in the structure built for that starting simplex. This gives again $O(m \log n)$ query time, plus the time to output all matches. 
Proof of Theorem 4. It is sufficient to prove the theorem for similarities as the most general case.

Let $B$ be the background set and let $B^{\prime}=B \cap D$ be a set of at least two points cut out by the circular disc $D$. Then the Delaunay-triangulation of $B^{\prime}$ has a common edge with that of $B$. Indeed, such edges can be found easily: Let $D^{\prime} \subseteq D$ be the smallest circular disc that contains $B^{\prime}$; then there is some point $b_{1} \in B^{\prime}$ on the boundary of $D^{\prime}$. Consider the family of discs contained in $D^{\prime}$ having $b_{1}$ on their boundary (with a common tangent with $D^{\prime}$ ); then there is a smallest such disc $D^{\prime \prime}$ which contains another point $b_{2}$ of $B^{\prime}$. Then $b_{1}, b_{2}$ are on the boundary of the disc $D^{\prime \prime}$, which does not contain any point of $B^{\prime}$ in its interior, and neither any point of $B$ (since $D^{\prime \prime} \subset D$ ), so $b_{1} b_{2}$ is an edge of the Delaunay-triangulations of $B$ and $B^{\prime}$.

Thus, given the pattern $A$, we determine the smallest disc $D_{A}$ containing $A$ (in $O(m \log m)$ time $)$ and select a point $a_{1}$ on the boundary of $D_{A}$. Then we compute for each point $a \in A$ the radius of the disc tangent to $D_{A}$ in $a_{1}$ and containing $a$ on its boundary, and choose as $a_{2}$ the point generating the disc of smallest radius (in time $O(m)$ ). Then $a_{1} a_{2}$ is a Delaunay-edge of $A$, and also a Delaunay-edge of any superset of $A$ that does not intersect $D_{A}$ in any further points. So in any occurrence of a similar copy of $A$ cut out by a circular window of $B$ the image $b_{1} b_{2}$ of $a_{1} a_{2}$ under that similarity must be a Delaunay-edge of $B$. There are only $O(n)$ Delaunay-edges, and a similarity in the plane is determined by the image of two points. So there are at most $O(n)$ possible matches, which can be found by constructing the Delaunay-triangulation in $O(n \log n)$ time.

\section{Acknowledgments}

The author thanks the referees for constructive and critical comments.

\section{References}

[AbF1] B.M. Ábrego, S. Fernández-Merchant: On the maximum number of equilateral triangles, I, Discrete Comput. Geom. 23 (2000), 129-135.

[AbF2] B.M. Ábrego, S. Fernández-Merchant: On the maximum number of congruent triangles among $n$ points in three-dimensional space, manuscript.

[AgS] P.K. Agarwal, M. Sharir: On the number of congruent simplices in a point set, SCG 01 (17th ACM Symp. Comput. Geom.), 2001, pp. 1-9.

[AkTT] T. Akutsu, H. Tamaki, T. Tokuyama: Distribution of distances and triangles in a point set and algorithms for computing the largest common point sets, Discrete Comput. Geom. 20 (1998), 307331.

[AlG] H. Alt, L.J. Guibas: Discrete geometric shapes: matching, interpolation, and approximation, in: Handbook of Computational Geometry, J.-R. Sack, J. Urrutia, eds., Elsevier, Amsterdam, 1999, pp. 121-153.

[Bo] L. Boxer: Point set pattern matching in 3-D, Pattern Recog. Lett. 17 (1996), 1293-1297.

$[\mathrm{BoH}]$ L. Boxer, R. Haralick: Even faster point set pattern matching in 3-D, Technical Report 2001-01 of the Department of Computer Science and Engineering, SUNY Buffalo.

[Br1] P. Braß: Exact point pattern matching and the number of congruent triangles in a three-dimensional pointset, ESA 2000 (European Symp. Algorithms), M. Paterson, ed., LNCS 1879, Springer-Verlag, Berlin, 2000, pp. 112-119. 
[Br2] P. Braß: On finding maximum-cardinality symmetric subsets, JCDCG 2000 (Jap. Conf. Discrete Comput. Geom.), J. Akiyama, M. Kano, M. Urabe, eds., LNCS 2098, Springer-Verlag, Berlin, 2001, pp. 106-112.

[BrK] P. Braß, C. Knauer: Testing the congruence of $d$-dimensional point sets, Proc. ACM Symp. Comput. Geom., ACM Press, New York, 2000, pp. 310-314.

[CaS] D. Cardoze, L.J. Schulman: Pattern matching for spatial point sets, Proc. 39th FOCS, IEEE Press, Piscataway, NJ, 1998, pp. 156-165.

[Ch1] N. Chevallier: Meilleures approximations d'un élément du tore $T^{2}$ et géométrie de la suite des multiples de cet élément, Acta Arith. 78 (1996), 19-35.

[Ch2] N. Chevallier: Geométrie des suites de Kronecker, Manuscripta Math. 94 (1997), 231-241.

[Ch3] N. Chevallier: Three distance theorem and grid graph, Discrete Math. 223 (2000), 355-362.

$\left[\mathrm{ClEG}^{+}\right]$K.L. Clarkson, H. Edelsbrunner, L. Guibas, M. Sharir, E. Welzl: Combinatorial complexity bounds for arrangements of curves and spheres, Discrete Comput. Geom. 5 (1990), 99-160.

[ElE] G. Elekes, P. Erdős: Similar configurations and pseudo grids, in Intuitive Geometry, K. Böröczky et al., eds., Colloq. Math. Soc. János Bolyai 63, North-Holland, Amsterdam, 1994, pp. 85-104.

[Er] P. Erdôs: On sets of distances of $n$ points, Amer. Math. Monthly 53 (1946), 248-250.

[ErHP] P. Erdôs, D. Hickerson, J. Pach: A problem of Leo Moser about repeated distances on the sphere, Amer. Math. Monthly 96 (1989), 569-575.

[ErP] P. Erdős, G. Purdy: Some extremal problems in geometry, IV, Proc. 7 th South-Eastern Conf. Combin. Graph Theory, Comput., Congressus Numerantium 17 Utilitas Math., Winnipeg, 1976, pp. 307-322.

[HuKK] D.P. Huttenlocher, K. Kedem, J.M. Kleinberg: On dynamic Voronoi diagrams and the minimum Hausdorff distance for point sets under Euclidean motion in the plane, Proc. ACM Symp. Comput. Geom., 1992, pp. 110-120.

[InV] P. Indyk, S. Venkatasubramanian: Approximate congruence in nearly linear time, Proc. ACM Symp. Discrete Algebra, 2000, pp. 354-360.

[KrB] M.J. van Krefeld, M.T. de Berg: Finding squares and rectangles in sets of points, $W G 1989$ (Graph-Theoretic Concepts Comput. Sci.), M. Nagl, ed., LNCS 411, Springer-Verlag, Berlin, 1989, pp. 341-355.

[LaR] M. Laczkovich, I.Z. Ruzsa: The number of homothetic subsets, in: The Mathematics of Paul Erdôs, Vol. II, R.L. Graham et al., eds., Algorithms and Combinatorics Series 14, Springer-Verlag, New York, 1997, pp. 294-302.

[Li] F.L. Liang: A short proof of the $3 d$ distance theorem, Discrete Math. 28 (1979), 325-326.

[Ma] J. Matoušek: Range searching with efficient hierarchical cuttings, Discrete Comput. Geom. 10 (1993), $157-182$.

[Na] M.B. Nathanson: Additive Number Theory: Inverse Problems and the Geometry of Sumsets, SpringerVerlag, New York, 1996.

[ReL] P.J. de Rezende, D.T. Lee: Point set pattern matching in $d$-dimensions, Algorithmica 13 (1995), $387-404$.

[Ru] W.J. Rucklidge: Lower bounds for the complexity of the graph of the Hausdorff distance as a function of transformation, Discrete Comput. Geom. 16 (1996), 135-153.

[SpST] J. Spencer, E. Szemerédi, W. Trotter: Unit distances in the euclidean plane, in: Graph Theory and Combinatorics, B. Bollobás, ed., Academic Press, London, 1984, pp. 293-304.

[Sz] L.A. Székely: Crossing numbers and hard Erdős problems in discrete geometry, Combin. Probab. Comput. 6 (1997), 353-358.

[Ta] G. Talenti: The standard isoperimetric theorem, in Handbook of Convex Geometry, P.M. Gruber, J.M. Wills, eds., Elsevier, Amsterdam, 1993, pp. 73-123.

[WeKA] G. Weber, L. Knipping, H. Alt: An application of point pattern matching in astronautics, J. Symbolic Comput. 17 (1994), 321-340.

Received March 7, 2001. Online publication October 29, 2002. 